\title{
Long noncoding RNA-p21 modulates cellular senescence via the Wnt/ $\beta$-catenin signaling pathway in mesenchymal stem cells
}

\author{
WENZHENG XIA $^{1 *}$, LEI ZHUANG ${ }^{2 *}$, XIA DENG $^{3}$ and MENG HOU ${ }^{3}$ \\ Departments of ${ }^{1}$ Neurosurgery, ${ }^{2}$ Hepatobiliary Surgery and ${ }^{3}$ Radiation Oncology, First Affiliated \\ Hospital, Wenzhou Medical University, Wenzhou, Zhejiang 325000, P.R. China
}

Received March 2, 2016; Accepted February 15, 2017

DOI: $10.3892 / \mathrm{mmr} .2017 .7430$

\begin{abstract}
Mesenchymal stem cell (MSC)-based therapies have demonstrated efficacy in animal models of cardiovascular diseases. However, MSCs decrease in quantity and quality with age, which reduces their capacity for damage repair. Long noncoding $(\operatorname{lnc})$ RNAs regulate gene transcription and the fate of post-transcriptional mRNA, affecting a broad range of age-associated physiological and pathological conditions, including cardiovascular disease and cancer cell senescence. However, the functional role of IncRNAs in stem cell senescence remains largely unknown. The present study isolated bone marrow-derived MSCs from young (8-week-old) and aged (18-month-old) male C57BL/6 mice. Cell proliferation was measured using a Cell Counting kit- 8 assay, and the secretion of vascular endothelial growth factor, basic fibroblast growth factor, hepatocyte growth factor and insulin-like growth factor was measured by ELISA. Western blotting was performed to investigate $\beta$-catenin protein expression. Oxidative stress was evaluated by detecting reactive oxygen species, and the activity of superoxide dismutase and malondialdehyde. MSCs isolated from aged mice demonstrated reduced proliferation and paracrine signaling, and increased oxidative stress and expression of lincRNA-p21compared with MSCs from younger mice. Silencing lincRNA-p21 in aged MSCs using small interfering RNA (siRNA) enhanced cell growth and paracrine function, and decreased oxidative stress. These results were reversed when $\beta$-catenin expression was silenced using siRNA. In conclusion, lincRNA-p21 may serve a role in MSC senescence, and silencing lincRNA-p21 may rejuvenate MSCs by interacting with the Wnt/ $\beta$-catenin signaling pathway. Targeting lincRNA-p21 may therefore have
\end{abstract}

Correspondence to: Dr Meng Hou, Department of Radiation Oncology, First Affiliated Hospital, Wenzhou Medical University, 2 Fuxue Lane, Wenzhou, Zhejiang 325000, P.R. China

E-mail: 244517813@qq.com

*Contributed equally

Key words: mesenchymal stem cell, senescence, long noncoding RNA-p21, Wnt/ $\beta$-catenin signaling pathway, oxidative stress important therapeutic implications for restoring endogenous MSCs in aged individuals.

\section{Introduction}

Myocardial infarction leads to a permanent loss of cardiomyocytes and results in pathological remodeling (1). Despite advances in the clinical treatment of heart failure, ischemia/reperfusion injury remains a major cause of mortality in developed countries (2). Numerous studies have demonstrated that autologous transplantation of bone marrow-derived mesenchymal stem cells (BM-MSCs) has potential for repairing and regenerating cardiomyocytes, as well as restoring heart function (3-5). However, clinical and animal studies have reported that MSCs derived from older donors have a reduced regenerative potential compared with those from younger donors. As myocardial infarction is more common in older individuals, this may limit the benefits of treatment with MSCs (6). Long noncoding RNAs (lncRNAs), located in the nucleus and cytosol of cells, have been demonstrated to be integral regulatory features in the genome (7). They have been revealed to regulate numerous biological processes, including transcription, differentiation, tissue development and tumorigenesis (8-11). LincRNA-p21 is involved in regulating tissue development and certain cellular functions, including proliferation, apoptosis, DNA damage and endoplasmic reticulum stress $(12,13)$. With respect to senescence, lincRNA-p21 is transcriptionally induced by $\mathrm{p} 53$, its expression is increased in senescent cells and it represses the translation of the proteins $\beta$-catenin and JunB, which promote cell proliferation (14). However, the biological function of lncRNAs in stem cell senescence remains to be fully elucidated. LncRNAs regulate diverse biological processes by forming lncRNA-protein and lncRNA-microRNA complexes that control gene expression and function (7). The Wnt/ $\beta$-catenin signaling network is closely associated with stem cell self-renewal and differentiation (15). Cytosolic $\beta$-catenin is translocated to the nucleus in the presence of Wnt activity, where it interacts with transcription factors to regulate genes associated with stem cell self-renewal, proliferation and differentiation (16). Wnt/ $\beta$-catenin signaling has previously been demonstrated to be involved in the rejuvenation of hematopoietic stem cells, by increasing their regenerative capacity (17). $\beta$-catenin was initially identified as a direct transcriptional target of 
lincRNA-p21, thereby regulating apoptosis, DNA damage and endoplasmic reticulum stress. In addition, a recent study demonstrated that lincRNA-p21 inhibited $\beta$-catenin signaling, thereby attenuating the viability, self-renewal and glycolysis of cancer stem cells (CSCs) (18). Based on the role of lincRNA-p21-regulated $\beta$-catenin signaling in stem cell senescence, the present study aimed to determine if modulating lincRNA-p21 activated $\beta$-catenin signaling and rejuvenated aged MSCs.

Reactive oxygen species (ROS) are additionally involved in inducing senescence under physiological and pathological conditions (19). A previous study demonstrated that ROS decreased the proliferative capacity and pluripotency of aged MSCs (20), and a recent report observed that apoptosis caused increased lincRNA-p21 expression and ROS-associated DNA damage (21). Endoplasmic reticulum stress induced by lincRNA-p21has been suggested to account for its effects on the apoptosis and proliferation of hepatocellular carcinoma cells (13). The current study investigated whether interfering with lincRNA-p21 may attenuate oxidative stress and promote the rejuvenation of aged MSCs.

\section{Materials and methods}

Animals. BM-MSCs were isolated from young (8-week-old) and aged (18-month-old) male C57BL/6 mice $(\mathrm{n}=12$, per age group), as described previously (22). Mice were purchased from the laboratory animals center of Wenzhou Medical University (Wenzhou, China). The animals were maintained on a 12 -h light/dark cycle, at $21 \pm 2^{\circ} \mathrm{C}$ and with a relative humidity of $30-70 \%$. Food and water was freely available throughout. All procedures were approved by the Animal Care and Use Committee of Wenzhou Medical University, and complied with the Guidelines for the Care and Use of Laboratory Animals, published by the National Academy Press (23).

Reagents. Dulbecco's modified Eagle's medium (DMEM) and fetal bovine serum (FBS) were purchased from HyClone (GE Healthcare Life Sciences, Logan, UT, USA), TRIzol ${ }^{\circledR}$ reagent was from Invitrogen; Thermo Fisher Scientific, Inc. (Waltham, MA, USA), and the Transcriptor First Stand cDNA Synthesis kit, FastStart Universal SYBR ${ }^{\circledR}$ Green Master (Rox) and X-treme GENE HP DNA transfection reagent were purchased from Roche Diagnostics (Basel, Switzerland). Rabbit monoclonal antibodies against $\beta$-catenin $(8480 ; 1 / 1,000)$ and $\beta$-actin $(4970 ; 1,1,000)$ were obtained from Cell Signaling Technology, Inc. (Danvers, MA, USA) and horseradish peroxidase-conjugated anti-rabbit secondary antibodies (sc-2357; 1/1,000) were purchased from Santa Cruz Biotechnology, Inc. (Dallas, TX, USA). Vascular endothelial growth factor (VEGF; RAB0509), basic fibroblast growth factor (bFGF; RAB0184), hepatocyte growth factor (HGF; RAB0214) and insulin-like growth factor (IGF; RAB0229) ELISA kits were purchased from Sigma-Aldrich (Merck Millipore, Darmstadt, Germany). Small interfering RNAs (siRNAs) targeting lincRNA-p21 (sequence: 5'-UGAAAAGAGCCGUGAGCUA-3'), $\beta$-catenin (sequence: 5'-CTCACTTGCAATAATTACAAA-3') and non-targeting siRNA-NT control (sequence: 5'-CTCUCC GAACGUGUCACGUTT-3') transcripts were purchased from
Thermo Fisher Scientific, Inc. Cell proliferation was assessed using Cell Counting kit-8 (CCK-8; HaiGene Technology Co., Ltd., Harbin, China). Superoxide Dismutase (SOD) Activity Colorimetric assay and Lipid Peroxidation (Malondialdehyde; MDA) assay kits were purchased from Abcam (Cambridge, UK).

Isolation, culture and characterization of MSCs. BM-MSCs were isolated using a standard protocol, as described previously (22). Briefly, mice were anaesthetized using isoflurane (5\% in induction chamber, $2 \%$ in mask; flow of oxygen, 1 litre/min; Santa Cruz Biotechnology, Inc.), and sacrificed by cervical dislocation. Bone marrow was isolated from the femur and tibia of mice by flushing with PBS. Adherent MSCs were propagated and maintained at $37^{\circ} \mathrm{C}$ and $5 \% \mathrm{CO}_{2}$ in high-glucose DMEM supplemented with $10 \% \mathrm{FBS}$ and $1 \%$ penicillin/streptomycin. Third-passage MSCs were used for experiments to avoid contamination with other cell types.

Cell proliferation assay. The rate of cell proliferation was estimated using aCCK-8 assay, according to the manufacturer's protocol. Briefly, cells $\left(1 \times 10^{5}\right.$ cells/well) cultured in 96-well plates for 1, 3, 5 or 7 days were incubated with CCK-8 solution for $1 \mathrm{~h}$ at $37^{\circ} \mathrm{C}$, following which the absorbance of each well at a wavelength of $450 \mathrm{~nm}$ was recorded.

Evaluation of VEGF, bFGF, HGF and IGF levels. The concentrations of VEGF, bFGF, HGF and IGF secreted by MSCs were assessed by ELISA, according to the manufacturer's protocol and as described previously (22).

Reverse transcription-quantitative polymerase chain reaction $(R T-q P C R)$. Gene expression levels were analyzed by RT-qPCR. Briefly, total cellular RNA was isolated using TRIzol and reverse transcribed using a Transcriptor First Stand cDNA Synthesis kit, according to the manufacturer's protocol. qPCR was performed using the FastStart Universal SYBR Green Master (Rox). The following primers were used: VEGF, forward, 5'-CTGTGTGCCGCTGATGCG CT-3' and reverse, 5'-TCGCCCTCCGGACCCAAAGT-3'; bFGF forward, 5'-AGATCATGCTTCCACCTCGT-3' and reverse, 5'-TGGGTCCCTTTCACTTTGCC-3; HGF forward, 5'-GGCCATGAATTTGACCTCTATGAA-3' and reverse, 5'-TTCAACTTCTGAACACTGAGGAAT-3'; IGF, forward, 5'-TTTCAACAAGCCCACAGGGT-3' and reverse, 5'-TTG AGGGGTGCGCAATACAT-3; GAPDH, forward, 5'-GGA GCCAAAAGGGTCATCAT-3' and reverse 5'-GTGATG GCATGGACTGTGGT-3'; lincRNA-p21, forward, 5'-CCT GTCCACTCGCTTTC-3' and reverse, 5'-GGAACTGGA GACGGAATGTC-3'. The thermocycling conditions were as follows: 40 cycles of amplification at $95^{\circ} \mathrm{C}$ for $15 \mathrm{sec}$, $64^{\circ} \mathrm{C}$ for $20 \mathrm{sec}$ and $72^{\circ} \mathrm{C}$ for $25 \mathrm{sec}$. The quantitation cycle $(\mathrm{Cq})$ was set within the exponential phase of the $\mathrm{PCR}$ reaction, and the $\Delta \mathrm{Cq}$ value for each target gene was calculated by subtracting the $\mathrm{Cq}$ value for the internal control GAPDH, from that of the target gene. Relative gene expression levels were calculated by comparing the $\Delta \mathrm{Cq}$ values between control and experimental conditions for each target PCR using the following equation: Relative gene

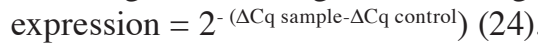


Knockdown of gene expression using siRNA. MSCs were transfected using the X-treme GENE HP DNA Transfection reagent, according to the manufacturer's protocol. Briefly, MSCs were cultured in6-well plates $\left(1 \times 10^{6}\right.$ cells/well $)$, treated with the transfection reagent at a reagent-to-siRNA weight ratio of $3: 1$ for $20 \mathrm{~min}$ at $37^{\circ} \mathrm{C}$, followed by the addition of a mixture containing $100 \mathrm{nM}$ siRNA and incubation in $2 \mathrm{ml}$ culture medium for $48 \mathrm{~h}$. Scrambled non-target-specific siRNA (siRNA-NT) served as a control. The transfection efficiency of siRNA-lincRNA-p 21 and siRNA- $\beta$-catenin was determined by RT-qPCR and western blotting, respectively.

Western blot analysis. Western blotting was performed as described previously (25). Briefly, cells were washed twice with ice-cold PBS and ruptured with lysis buffer. The lysates were centrifuged at $4^{\circ} \mathrm{C}$ for $5 \mathrm{~min}$ at $12,000 \mathrm{x} \mathrm{g}$ and the resulting supernatant contained total cellular protein. Proteins were quantified using bicinchoninic acid assay, and $20 \mu \mathrm{g}$ total protein of each sample was resolved by $10 \%$ SDS-PAGE and transferred onto polyvinylidene difluoride membranes. Membranes were blocked for $1 \mathrm{~h}$ with $5 \%$ skim milk in TBS containing $0.1 \%$ Tween-20 and incubated overnight at $4{ }^{\circ} \mathrm{C}$ with primary antibodies. The membranes were subsequently washed, incubated for $1 \mathrm{~h}$ with appropriate horseradish peroxidase-conjugated secondary antibodies and developed using BeyoECL Plus chemiluminescent substrate (Beyotime Institute of Biotechnology, Jiangsu, China). The stained protein bands were visualized using Bio-RadChemiDoc ${ }^{\mathrm{TM}}$ XRS equipment (Bio-Rad Laboratories, Inc., Hercules, CA, USA) and quantified using Quantity One software version 4.5.2 (Bio-Rad Laboratories, Inc.).

ROS measurement. Levels of intracellular ROS were determined using 2,7-dichlorodihydrofluorescein diacetate, supplied in the Reactive Oxygen Species Assay kit (Beyotime Institute of Biotechnology) according to the manufacturer's protocol. The fluorescence intensity of the cells was measured using a fluorescence spectrophotometer, with excitation and emission wavelengths of 488 and $525 \mathrm{~nm}$, respectively.

SOD activity. SOD activity in MSCs was determined using a colorimetric assay kit according to the manufacturer's protocol. Briefly, protein was isolated from MSCs using lysis buffer and SOD activity was measured in $10 \mu \mathrm{g}$ of total protein extract. Absorbance was measured at a wavelength of $450 \mathrm{~nm}$.

MDA lipid peroxidation assay. Lipid peroxidation was monitored using an assay kit to measure the formation of MDA, according to the manufacturer's protocol. Briefly, MSCs $\left(1 \times 10^{6}\right.$ cells) were homogenized on ice in $300 \mu 1$ MDA lysis buffer (with $3 \mu 1$ 100Xbutylated hydroxytoluene) and centrifuged $\left(13,000 \mathrm{x} \mathrm{g}\right.$ for $10 \mathrm{~min}$ at $\left.4^{\circ} \mathrm{C}\right)$ to remove insoluble material. The supernatant $(200 \mu \mathrm{l})$ was added to $600 \mu \mathrm{l}$ thiobarbituric acid and incubated at $95^{\circ} \mathrm{C}$ for $60 \mathrm{~min}$. The samples were cooled to room temperature in an ice bath for $10 \mathrm{~min}$ and the absorbance at a wavelength of $532 \mathrm{~nm}$ was measured spectrophotometrically.

Statistical analysis. Data are presented as the mean \pm standard deviation. Differences among groups were analyzed by one-way analysis of variance, followed by Tukey's test for multiple comparisons. Analyses between two groups were performed using Student's t-test. Statistical tests were performed using SPSS software version 19.0 (IBM SPSS, Armonk, NY, USA). $\mathrm{P}<0.05$ was considered to indicate a statistically significant difference.

\section{Results}

Effects of age on MSC proliferation and paracrine ability. As senescence is associated with reduced cellular function, the present study compared the proliferative and paracrine abilities of aged and young MSCs. The self-renewal potential of young and aged MSCs was examined by CCK-8 assay, which confirmed low rates of proliferation in aged MSCs after 1, 3, 5 and 7 days of culture (Fig. 1A). The expression and secretion of VEGF, bFGF, HGF, and IGF was also investigated by RT-qPCR and ELISA, respectively. mRNA expression levels of all four factors were significantly reduced in aged compared with younger MSCs (Fig. 1B-E) and the corresponding protein levels in the culture medium were additionally significantly decreased in aged compared with younger MSCs (Fig. 1F-I).

Effects of age on lincRNA-p21 expression, cellular oxidative stress and the Wnt/ $\beta$-catenin signaling pathway. lincRNA-p21 expression levels were significantly greater in aged compared with younger MSCs (8.67 \pm 0.35 vs. $1.00 \pm 0.00 ; \mathrm{P}<0.05$; Fig. 2A). lincRNA-p21 has previously been demonstrated to reduce $\beta$-catenin protein levels in CSCs (18). In the present study, $\beta$-catenin protein expression levels were decreased in aged compared with younger MSCs $(1.00 \pm 0.00$ vs. $4.61 \pm 0.41$; $\mathrm{P}<0.05$; Fig. 2B and C). To determine whether cellular senescence involved oxidative stress, the present study investigated ROS generation, SOD activation and lipid peroxidation by MDA assay. Senescence was associated with a significantly increased generation of ROS (100.00 \pm 0.00 vs. $13.58 \pm 1.60 \%$; $\mathrm{P}<0.05$; Fig. 2D), significantly reduced SOD activity $(1.00 \pm 0.00$ vs. 3.33 $\pm 0.28 ; \mathrm{P}<0.05$; Fig. $2 \mathrm{E})$ and significantly increased MDA activity $(100.00 \pm 0.00$ vs. $11.40 \pm 0.96 \%$; $\mathrm{P}<0.05$; Fig. $2 \mathrm{~F})$ compared with younger MSCs.

Effect of lincRNA-p21 knockdown on rejuvenation of aged MSCs. The role of lincRNA-p21 in rejuvenating aged MSCs was further investigated by knockdown of endogenous lincRNA-p21 by siRNA. LincRNA-p21 expression levels were significantly reduced by transfection with lincRNA-p21 siRNA compared with siRNA-NT (10.47 \pm 1.00 vs. $100.00 \pm 0.00 \%$; $\mathrm{P}<0.05$; Fig. 3A). Knockdown of lincRNA-p21 was associated with significantly increased expression of $\beta$-catenin $(3.92 \pm 0.29$ vs. $1.00 \pm 0.00 ; \mathrm{P}<0.05$; Fig. $3 \mathrm{~B}$ and $\mathrm{C}$ ) and significantly increased proliferation of aged MSCs (Fig. 3D) compared with the siRNA-NT control. In addition, inhibition of lincRNA-p21 increased the paracrine ability of aged MSCs, which was indicated by significantly increased secretion of VEGF, bFGF, HGF and IGF (Fig. 3E-H), decreased generation of ROS (12.22 \pm 1.07 vs. $100.00 \pm 0.00 \%$; $\mathrm{P}<0.05$; Fig. 3I), increased SOD activity (3.25 \pm 0.17 vs. $1.00 \pm 0.00 ; \mathrm{P}<0.05$; Fig. $3 \mathrm{~J})$ and reduced MDA activity $(11.34 \pm 1.00$ vs. $100.00 \pm 0.00 \%$; P<0.05; Fig. $3 \mathrm{~K})$, compared with siRNA-NT. In addition, siRNA-lincRNA-p21 transfected MSCs were compared with young MSCs; no 

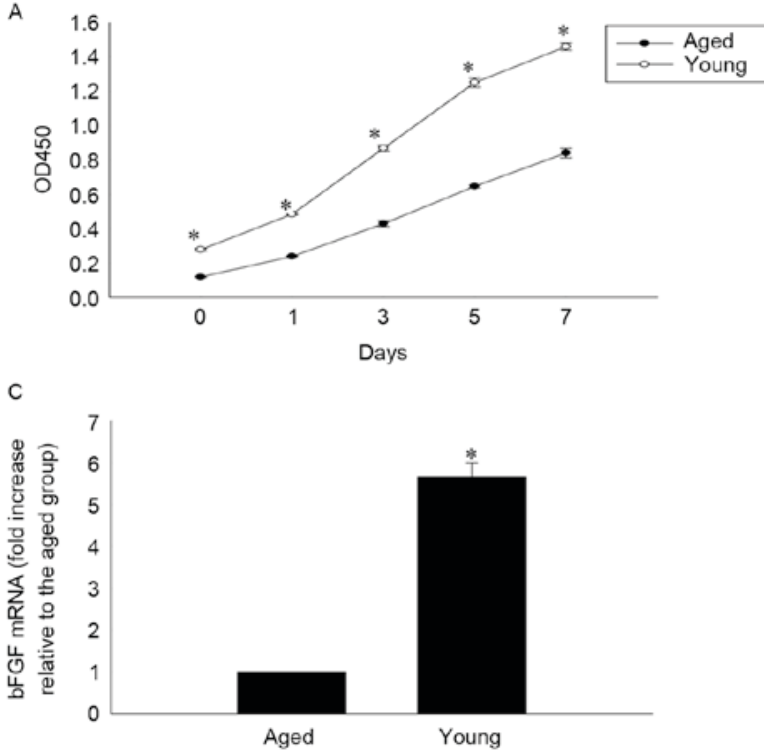

E

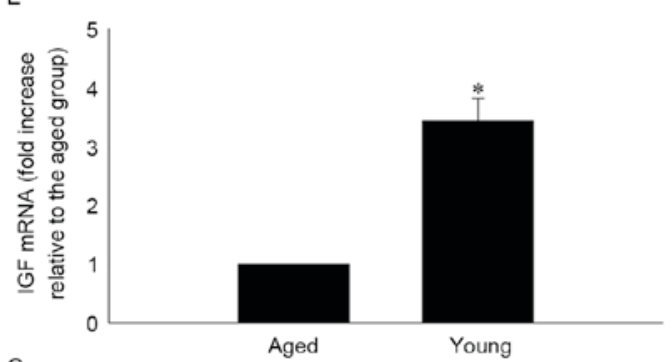

G
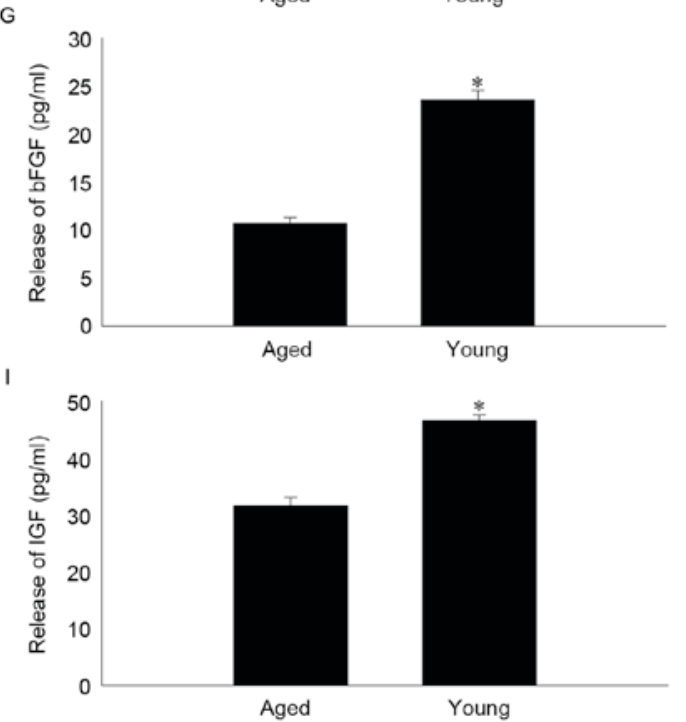

B

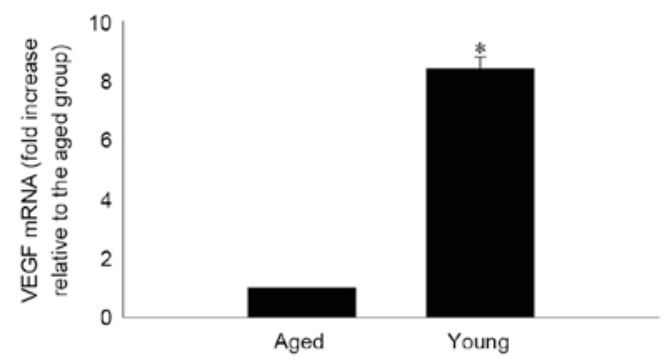

D
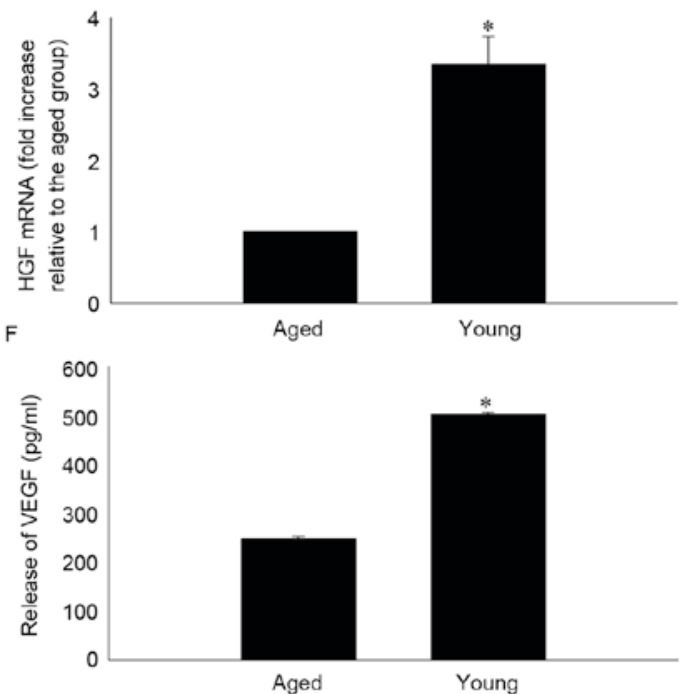

H

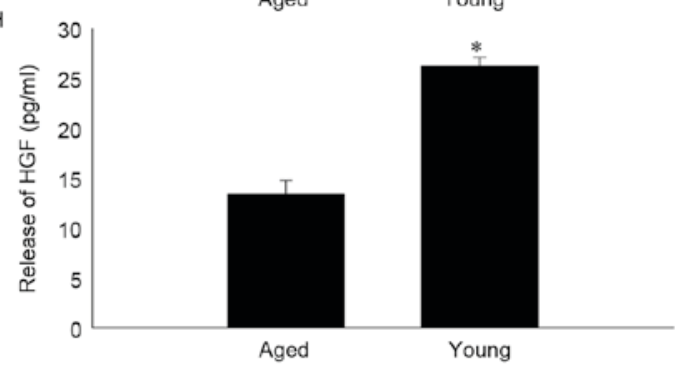

Figure 1. Age-impaired MSC proliferation and paracrine ability. (A) Cell proliferation curves of MSCs, as determined by Cell Counting kit-8 assay in young and aged MSCs at 1,3,5 and 7 days. Relative mRNA levels of (B) VEGF, (C) bFGF, (D) HGF and (E) IGF analyzed by reverse transcription-quantitative polymerase chain reaction in cultures of young and aged MSCs. Secretion of (F) VEGF, (G) bFGF, (H) HGF and (I) IGF into the culture medium of young and aged MSCs, as analyzed by ELISA. Data are presented as the mean \pm standard deviation from three independent experiments. "P $<0.05$ vs. aged MSCs. MSCs, mesenchymal stem cells; VEGF, vascular endothelial growth factor; bFGF, basic fibroblast growth factor; HGF, hepatocyte growth factor; IGF, insulin-like growth factor; OD, optical density.

significant difference between the siRNA-lincRNA-p21 group and the young group in proliferation and paracrine ability was observed (data not shown).

Role of the Wnt/ $\beta$-catenin signaling pathway in lincRNAp21-induced effects on MSCs. The present study further investigated the mechanism underlying the effect of lincRNA-p21on the rejuvenation of aged MSCs by silencing $\beta$-catenin using siRNA. $\beta$-catenin protein expression levels were significantly reduced in cells transfected with siRNA- $\beta$-catenin compared with siRNA-NT control $(21.77 \pm 1.37$ vs. $100.00 \pm 0.00 \%$; $\mathrm{P}<0.05$; Fig. $4 \mathrm{~A}$ and $\mathrm{B}$ ). Knockdown of lincRNA-p21 increased the proliferation of aged MSCs and increased their paracrine ability; these effects were abolished by silencing 

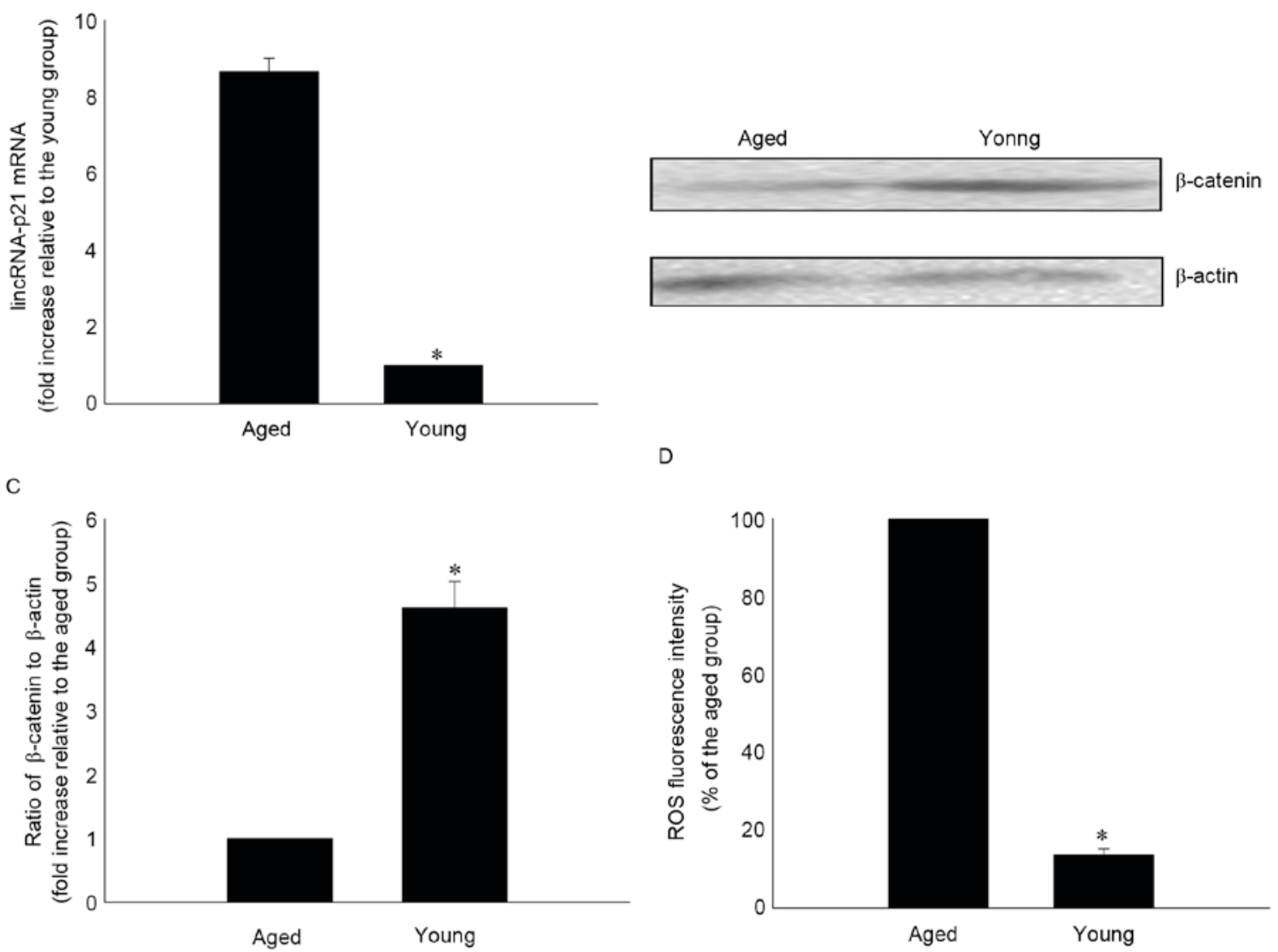

$\mathrm{E}$
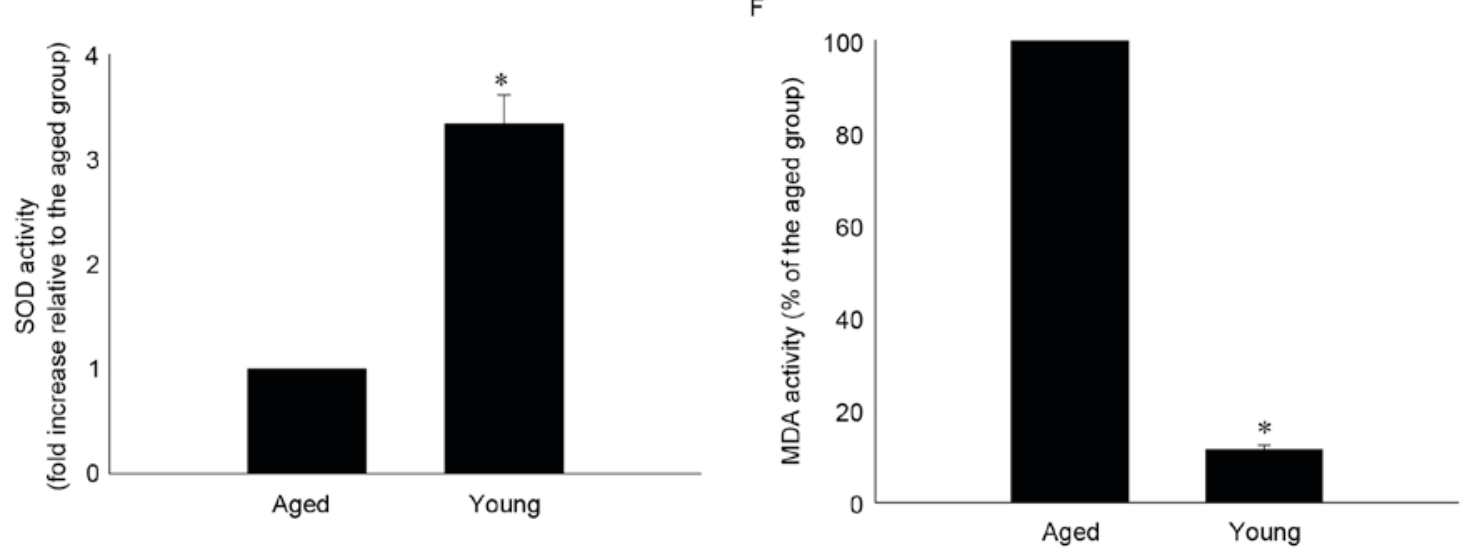

Figure 2. Effect of age on the expression of lincRNA-p21, cellular oxidative stress and the Wnt//-catenin pathway. (A) lincRNA-p21 mRNA expression levels analyzed by reverse transcription-quantitative polymerase chain reaction in aged and young MSCs. (B) Image and (C) quantification of western blotting for $\beta$-catenin protein expression levels in aged and young MSCs. (D) Intracellular ROS production in MSCs, as analyzed by fluorescence spectrophotometry. (E) SOD activity, as evaluated by colorimetric assay. (F) Lipid peroxidation, as evaluated by MDA formation. Data are presented as the mean \pm standard deviation of three independent experiments. "P<0.05 vs. aged MSCs. IncRNA, long noncoding RNA; MSCs, mesenchymal stem cells; ROS, reactive oxygen species; SOD, superoxide dismutase; MDA, malondialdehyde.

$\beta$-catenin (Fig. 4C-G). The decrease in oxidative stress associated with knockdown of lincRNA-p21 expression in aged MSCs was additionally reversed by silencing $\beta$-catenin, with increased generation of ROS (98.55 \pm 0.47 vs. $13.29 \pm 0.99 \%$; $\mathrm{P}<0.05$; Fig. $4 \mathrm{H})$, decreased SOD activity $(1.08 \pm 0.05$ vs. $3.29 \pm 0.11 ; \mathrm{P}<0.05$; Fig. 4I) and increased MDA activity $(98.77 \pm 0.76$ vs. $11.66 \pm 0.43 \%$; $\mathrm{P}<0.05$; Fig. $4 \mathrm{~J})$, compared with siRNA-lincRNA-p21-transfected cells.

\section{Discussion}

MSCs isolated from adult bone marrow are an excellent source for therapeutic stem cell development (26). However, MSCs undergo age-associated alterations, including reduced proliferation and paracrine signaling (27), which decrease their capacity for damage repair (28). Numerous strategies have been investigated to overcome these limitations, and certain 
$\mathbf{A}$
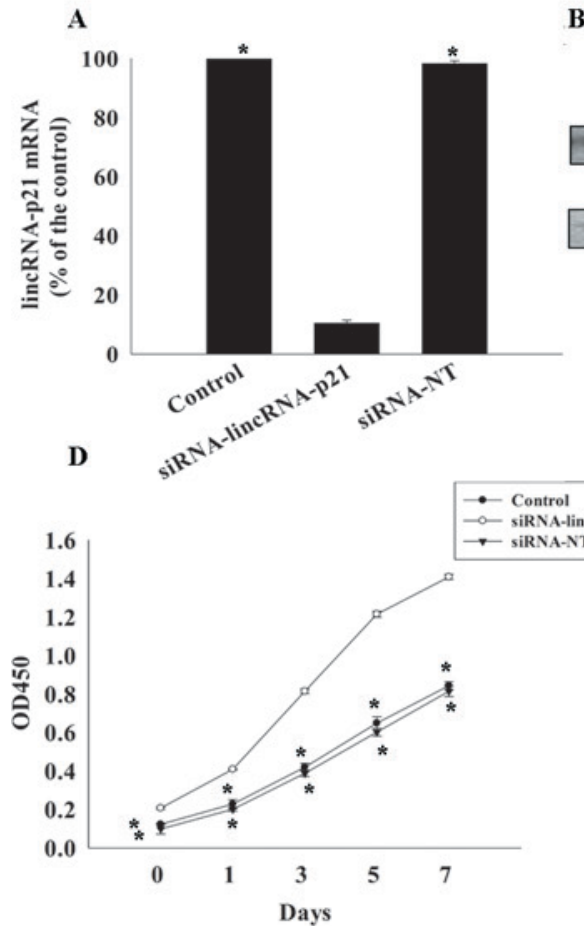

G
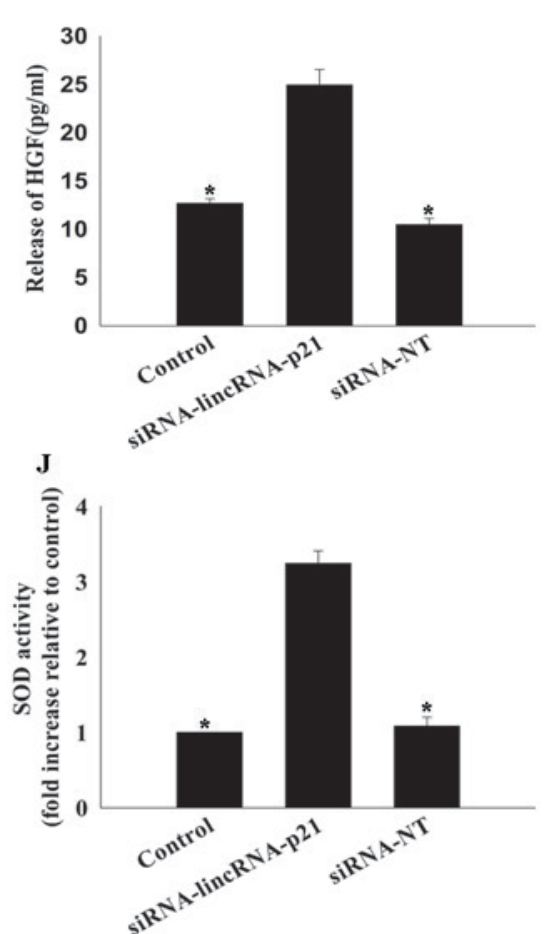

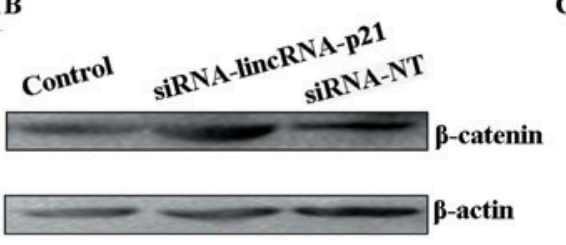

C

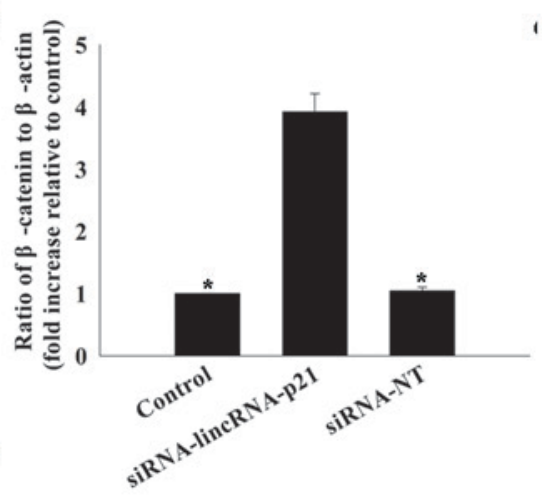

$\mathbf{E}$

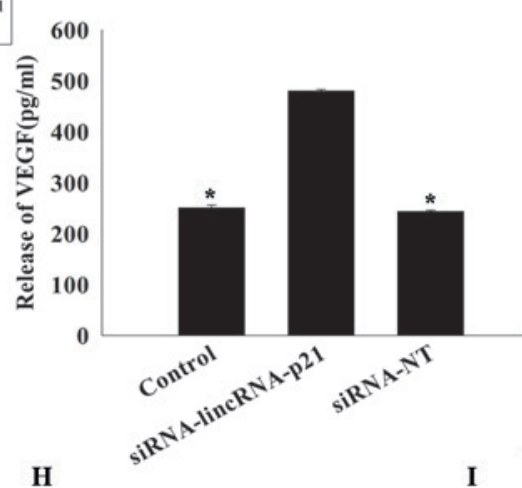

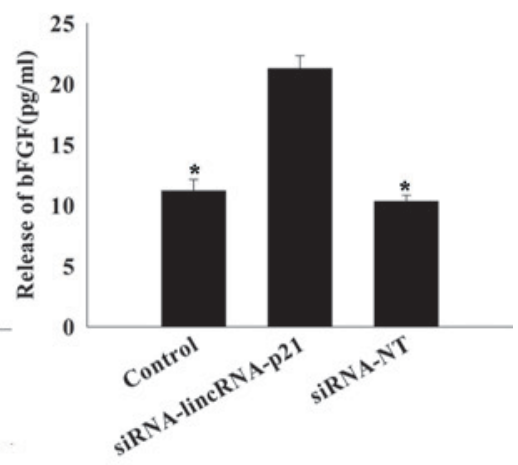
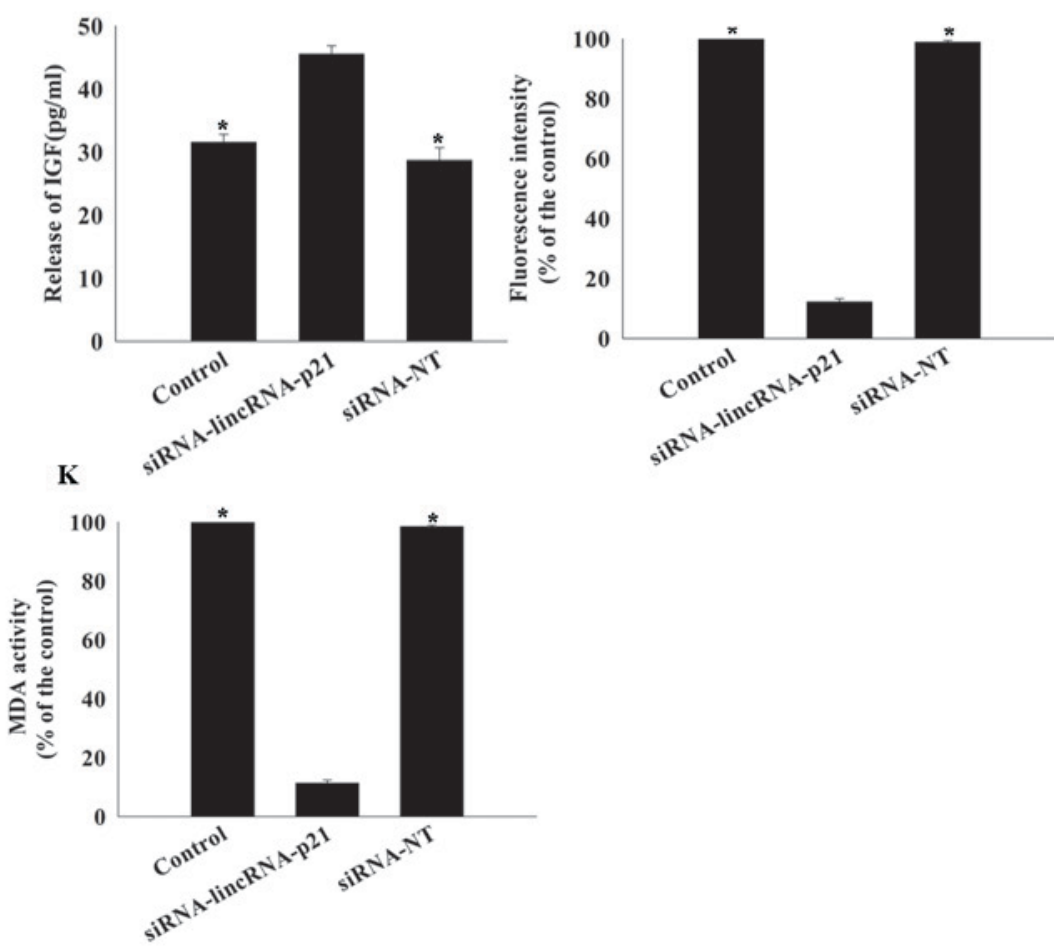

Figure 3. Effects of lincRNA-p21 silencing on rejuvenation and reduced oxidative stress in aged MSCs. (A) Reverse transcription-quantitative polymerase chain reaction analysis of lincRNA-p21expression in untransfected MSCs, and MSCs transfected with lincRNA-p21-specific siRNA or control siRNA-NT. (B) Image and (C) quantification of western blotting for $\beta$-catenin protein expression levels in untransfected MSCs, and MSCs transfected with lincRNA-p21-specific siRNA or siRNA-NT. (D) Cell proliferation curves of untransfected MSCs, and MSCs transfected with siRNA-lincRNA-p21 orsiRNA-NT. Concentrations of (E) VEGF, (F) bFGF, (G) HGF and (H) IGF in the culture medium of aged untransfected MSCs, and MSCs transfected with siRNA-lincRNA-p21 or siRNA-NT. (I) Intracellular ROS production, (J) SOD activity and (K) MDA activity were measured in aged untransfected MSCs, and aged MSCs transfected with siRNA-lincRNA-p21 orsiRNA-NT. Data are presented as the mean \pm standard deviation from three independent experiments. "P $<0.05$ vs. siRNA-lincRNA-p21. IncRNA, long noncoding RNA; MSCs, mesenchymal stem cells; siRNA, small interfering RNA; siRNA-NT, non-target-specific small interfering RNA; VEGF, vascular endothelial growth factor; bFGF, basic fibroblast growth factor; HGF, hepatocyte growth factor; IGF, insulin-like growth factor; ROS, reactive oxygen species; SOD, superoxide dismutase; MDA, malondialdehyde; OD, optical density.

of these have resulted in marked improvements in cardiac function, particularly in a rodent model of acute myocardial infarction (22). However, the mechanisms underlying MSC senescence remain unclear. The results of the present study 


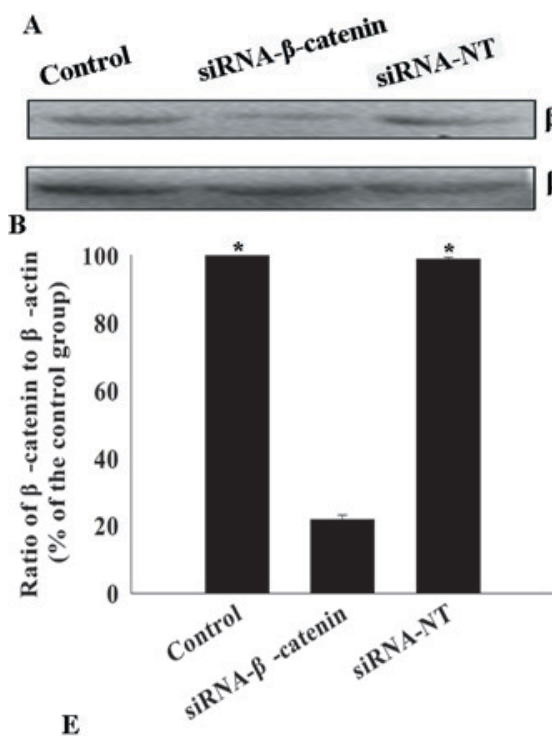

\section{$\beta$-catenin}
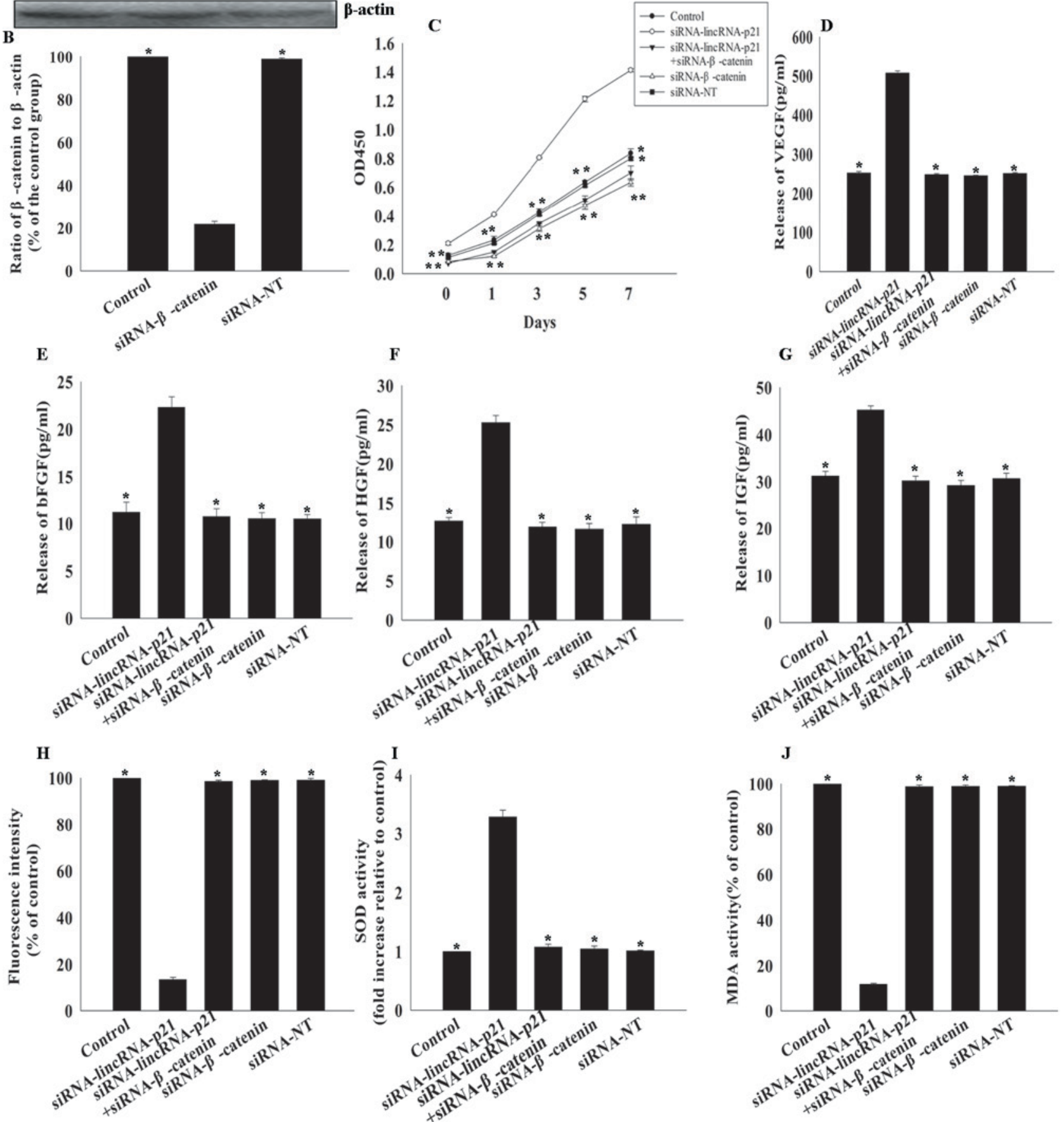

Figure 4. Effects of lincRNA-p21 on MSC rejuvenation via the Wnt/ $\beta$-catenin pathway. (A) Image and (B) quantification of western blotting for $\beta$-catenin protein expression levels in untransfected MSCs, and MSCs transfected with $\beta$-catenin-siRNA or siRNA-NT. " $\mathrm{P}<0.05$ vs. siRNA- $\beta$-catenin. (C) Cell proliferation curves, as determined by Cell Counting kit-8 assay, of untransfected MSCs, and MSCs transfected withlincRNA-p21-siRNA, $\beta$-catenin-siRNA, lincRNA-p21-siRNA + $\beta$-catenin-siRNA or siRNA-NT. "P<0.05 vs. siRNA-lincRNA-p21. Concentrations of (D) VEGF, (E) bFGF, (F) HGF and (G) IGF in culture medium of untransfected MSCs, and MSCs transfected with lincRNA-p21-siRNA, $\beta$-catenin-siRNA, lincRNA p21-siRNA + $\beta$-catenin-siRNA or siRNA-NT. "P<0.05 vs. siRNA-lincRNA-p21. (H) Intracellular ROS production, (I) SOD activity and (J) MDA activity were measured in untransfected MSCs, and MSCs transfected with lincRNA-p21-siRNA, $\beta$-catenin-siRNA, lincRNA-p21-siRNA + $\beta$-catenin-siRNA or siRNA-NT. "P<0.05 vs. siRNA-lincRNA-p21. Data are presented as the mean \pm standard deviation from three independent experiments. IncRNA, long noncoding RNA; MSCs, mesenchymal stem cells; siRNA, small interfering RNA; siRNA-NT, non-target-specific small interfering RNA; VEGF, vascular endothelial growth factor; bFGF, basic fibroblast growth factor; HGF, hepatocyte growth factor; IGF, insulin-like growth factor; ROS, reactive oxygen species; SOD, superoxide dismutase; MDA, malondialdehyde; OD, optical density.

identified lincRNA-p21 as an important mediator of cellular senescence and suggested that modification of lincRNA-p21 expression rejuvenated aged MSCs via the Wnt/ $\beta$-catenin signaling pathway. 
lncRNAs are non-protein-coding RNAs that are $>200$ nucleotides; increasing evidence has implicated them in the regulation of various cellular functions and development processes (29). lncRNAs differ widely in length and function, and regulate gene transcription and the fate of post-transcriptional mRNA (18). Collectively, IncRNAs affect a broad range of age-associated physiological and pathological conditions (30). The results of the current study demonstrated that lincRNA-p21 expression was greater in MSCs from aged mice compared with those from younger mice, and this high level of expression was accompanied by reduced cell proliferation and impaired paracrine ability. The role of lincRNA-p21 in MSC senescence was confirmed by silencing with siRNA, which led to increased cell proliferation and paracrine ability, and reduced oxidative stress, indicating rejuvenation of aged MSCs.

Various members of the Wnt family are expressed in stem cells (31). Wnt3a/ $\beta$-catenin are associated with canonical Wnt signaling and have been widely investigated in relation to stem cell senescence (32), whereas $\beta$-catenin has been previously proposed to increase hematopoietic cell self-renewal (17). The Wnt/ $\beta$-catenin network represents a potential target for MSC rejuvenation and has been confirmed to have an important role in restoring MSC myogenic differentiation by increasing $\beta$-catenin bioavailability (16). In the current study, the Wnt/ $\beta$-catenin signaling pathway appeared to be inhibited in aged MSCs, indicating that it may be involved in the rejuvenation process. LncRNAs regulate diverse biological processes through the formation of IncRNA-protein and IncRNA-microRNA complexes, thereby controlling gene expression and function (7). LincRNA-p21 directly downregulates $\beta$-catenin protein levels in various cell types, and vector-delivered lincRNA-p21 preferentially blocked the activation of Wnt/ $\beta$-catenin signaling in CSCs. The viability, self-renewal and tumorigenicity of CSCs were compromised by lincRNA-p21 overexpression (18). The present study observed that the Wnt/ $\beta$-catenin signaling pathway was associated with the underlying mechanism of lincRNA-p21, and silencing lincRNA-p21 restored $\beta$-catenin expression in aged MSCs. In addition, the current study demonstrated that MSC rejuvenation induced by silencing lincRNA-p21 was abolished by silencing $\beta$-catenin, indicating that lincRNA-p21-associated aging may involve inhibition of the Wnt/ $\beta$-catenin signaling pathway.

Oxidative stress is generally associated with induction of cell aging (33). It is accompanied by ROS generation, increased oxidant enzyme activity and diminished antioxidant enzyme activity (34). Cellular senescence is associated with metabolic function disorders and recent studies have identified roles for multiple lncRNA pathways in the control of metabolic function (7). LincRNA-p21 is associated with cellular DNA damage and endoplasmic reticulum stress under oxidative stress (13). Oxidative stress diverts $\beta$-catenin from the transcriptional pathways of the T-cell factor/lymphoid enhancer factor family to forkhead box $\mathrm{O}$, indicating that oxidative stress-associated $\beta$-catenin downregulation may be involved in aging in mice (35). The results of the current study demonstrated that oxidative stress was increased in aged MSCs, which was associated with high lincRNA-p21 and low $\beta$-catenin expression levels, and was supported by the observation that silencing lincRNA-p21 decreased oxidative stress, and that this effect was reversed by silencing $\beta$-catenin.
In conclusion, the results of the present study indicated that lincRNA-p21 is involved in MSC senescence. Interfering with lincRNA-p21 expression may allow the rejuvenation of aged MSCs by regulation of oxidative stress via the Wnt $/ \beta$-catenin signaling pathway. The demonstration that MSC senescence was delayed and regenerative properties enhanced has important therapeutic implications for vascular disorders. Targeting lincRNA-p21 expression in aged MSCs may be a useful strategy in cell transplantation-based repair and regeneration of peripheral and coronary vascular lesions.

\section{Acknowledgements}

The present study was supported by the National Natural Science Foundation of China (grant no. 81500261; to M.H.) and the Science and Technology Planning Project of Wenzhou (grant no. Y20160125; to M.H.).

\section{References}

1. Wollert KC, Meyer GP, Lotz J, Ringes-Lichtenberg S, Lippolt P, Breidenbach C, Fichtner S, Korte T, Hornig B, Messinger D, et al: Intracoronary autologous bone-marrow cell transfer after myocardial infarction: The BOOST randomised controlled clinical trial. Lancet 364: 141-148, 2004.

2. Stamm C, Westphal B, Kleine HD, Petzsch M, Kittner C, Klinge $\mathrm{H}$, Schümichen $\mathrm{C}$, Nienaber $\mathrm{CA}$, Freund $\mathrm{M}$ and Steinhoff G: Autologous bone-marrow stem-cell transplantation for myocardial regeneration. Lancet 361: 45-46, 2003.

3. Orlic D, Kajstura J, Chimenti S, Limana F, Jakoniuk I, Quaini F, Nadal-Ginard B, Bodine DM, Leri A and Anversa P: Mobilized bone marrow cells repair the infarcted heart, improving function and survival. Proc Natl Acad Sci USA 98: 10344-10349, 2001.

4. Orlic D, Kajstura J, Chimenti S, Jakoniuk I, Anderson SM, Li B, Pickel J, McKay R, Nadal-Ginard B, Bodine DM, et al: Bone marrow cells regenerate infarcted myocardium. Nature 410: 701-705, 2001.

5. Stamm C, Westphal B, Kleine HD, Petzsch M, Kittner C, Klinge $H$, Schümichen $C$, Nienaber CA, Freund $M$ and Steinhoff G: Autologous bone-marrow stem-cell transplantation for myocardial regeneration. Lancet 361: 45-46, 2003.

6. Dimmeler S and Leri A: Aging and disease as modifiers of efficacy of cell therapy. Circ Res 102: 1319-1330, 2008.

7. Zhao XY and Lin JD: Long noncoding RNAs: A new regulatory code in metabolic control. Trends Biochem Sci 40: 586-596, 2015.

8. Kornfeld JW and Brüning JC: Regulation of metabolism by long, non-coding RNAs. Front Genet 5: 57, 2014.

9. Lee JT: Epigenetic regulation by long noncoding RNAs. Science 338: 1435-1439, 2012.

10. Mercer TR and Mattick JS: Structure and function of long noncoding RNAs in epigenetic regulation. Nat Struct Mol Biol 20: 300-307, 2013.

11. Wang KC and Chang HY: Molecular mechanisms of long noncoding RNAs. Mol Cell 43: 904-914, 2011.

12. Blume CJ, Hotz-Wagenblatt A, Hüllein J, Sellner L, Jethwa A, Stolz T, Slabicki M, Lee K, Sharathchandra A, Benner A, et al: p53-dependent non-coding RNA networks in chronic lymphocytic leukemia. Leukemia 29: 2015-2023, 2015.

13. Yang N, Fu Y,Zhang H, Sima H,Zhu N and Yang G: LincRNA-p21 activates endoplasmic reticulum stress and inhibits hepatocellular carcinoma. Oncotarget 6: 28151-28163, 2015.

14. Yoon JH, Abdelmohsen K, Srikantan S, Yang X, Martindale JL, De S, Huarte $\mathrm{M}$, Zhan $\mathrm{M}$, Becker $\mathrm{KG}$ and Gorospe $\mathrm{M}$ : LincRNA-p21 suppresses target mRNA translation. Mol Cell 47: 648-655, 2012.

15. Paige SL, Osugi T, Afanasiev OK, Pabon L, Reinecke H and Murry CE: Endogenous Wnt/beta-catenin signaling is required for cardiac differentiation in human embryonic stem cells. PLoS One 5: e11134, 2010.

16. Brunt KR, Zhang Y, Mihic A, Li M, Li SH, Xue P, Zhang W, Basmaji S, Tsang K, Weisel RD, et al: Role of WNT/ $\beta$-catenin signaling in rejuvenating myogenic differentiation of aged mesenchymal stem cells from cardiac patients. Am J Pathol 181: 2067-2078, 2012 . 
17. Florian MC, Nattamai KJ, Dörr K, Marka G, Uberle B, Vas V, Eckl C, Andrä I, Schiemann M, Oostendorp RA, et al: A canonical to non-canonical Wnt signalling switch in haematopoietic stem-cell ageing. Nature 503: 392-396, 2013.

18. Wang J, Lei ZJ, Guo Y, Wang T, Qin ZY, Xiao HL, Fan LL, Chen DF, Bian XW, Liu J and Wang B: miRNA-regulated delivery of lincRNA-p21 suppresses $\beta$-catenin signaling and tumorigenicity of colorectal cancer stem cells. Oncotarget 6 : 37852-37870, 2015

19. Shi Y, Nikulenkov F, Zawacka-Pankau J, Li H, Gabdoulline R, $\mathrm{Xu} \mathrm{J}$, Eriksson S, Hedström E, Issaeva N, Kel A, et al: ROS-dependent activation of JNK converts p53 into an efficient inhibitor of oncogenes leading to robust apoptosis. Cell Death Differ 21: 612-623, 2014

20. Gharibi B, Farzadi S, Ghuman M and Hughes FJ: Inhibition of $\mathrm{Akt} / \mathrm{mTOR}$ attenuates age-related changes in mesenchymal stem cells. Stem Cells 32: 2256-2266, 2014.

21. Hall JR, Messenger ZJ, Tam HW, Phillips SL, Recio L and Smart RC: Long noncoding RNA lincRNA-p21 is the major mediator of UVB-induced and p53-dependent apoptosis in keratinocytes. Cell Death Dis 6: e1700, 2015.

22. Madonna R, Taylor DA, Geng YJ, De Caterina R, Shelat H, Perin EC and Willerson JT: Transplantation of mesenchymal cells rejuvenated by the overexpression of telomerase and myocardin promotes revascularization and tissue repair in a murine mode of hindlimb ischemia. Circ Res 113: 902-914, 2013.

23. Liu WH, Liu JJ, Wu J, Zhang LL, Liu F, Yin L, Zhang MM and Yu B: Novel mechanism of inhibition of dendritic cells maturation by mesenchymal stem cells via interleukin-10 and the JAK1/STAT3 signaling pathway. PLoS One 8: e55487, 2013.

24. Livak KJ and Schmittgen TD: Analysis of relative gene expression data using real-time quantitative PCR and the 2(-Delta Delta C(T)) method. Methods 25: 402-408, 2001

25. Hou M, Cui J, Liu J, Liu F, Jiang R, Liu K, Wang Y, Yin L, Liu W and Yu B: Angiopoietin-like 4 confers resistance to hypoxia/serum deprivation-induced apoptosis through PI3K/Akt and ERK1/2 signaling pathways in mesenchymal stem cells. PLoS One 9: e85808, 2014
26. Liang H, Hou H, Yi W, Yang G, Gu C, Lau WB, Gao E, Ma X, Lu Z, Wei X, et al: Increased expression of pigment epithelium-derived factor in aged mesenchymal stem cells impairs their therapeutic efficacy for attenuating myocardial infarction injury. Eur Heart J 34: 1681-1690, 2013.

27. Stenderup K, Justesen J, Clausen C and Kassem M: Aging is associated with decreased maximal life span and accelerated senescence of bone marrow stromal cells. Bone 33: 919-926, 2003.

28. Zhang JH, Sampogna S, Morales FR and Chase MH: Age-related ultrastructural changes in hypocretinergic terminals in the brainstem and spinal cord of cats. Neurosci Lett 373: 171-174, 2005.

29. Batista PJ and Chang HY: Long noncoding RNAs: Cellular address codes in development and disease. Cell 152: 1298-1307, 2013.

30. Greco S, Gorospe M and Martelli F: Noncoding RNA in age-related cardiovascular diseases. J Mol Cell Cardiol 83: 142-155, 2015.

31. Malhotra S and Kincade PW: Wnt-related molecules and signaling pathway equilibrium in hematopoiesis. Cell Stem Cell 4: 27-36, 2009.

32. Nemeth MJ, Topol L, Anderson SM, Yang Y and Bodine DM: Wnt5a inhibits canonical Wnt signaling in hematopoietic stem cells and enhances repopulation. Proc Natl Acad Sci USA 104: 15436-15441, 2007.

33. Henson SM, Lanna A, Riddell NE, Franzese O, Macaulay R, Griffiths SJ, Puleston DJ, Watson AS, Simon AK, Tooze SA and Akbar AN: p38 signaling inhibits mTORC1-independent autophagy in senescent human $\mathrm{CD} 8^{+} \mathrm{T}$ cells. J Clin Invest 124 4004-4016, 2014.

34. Finkel $\mathrm{T}$ and Holbrook NJ: Oxidants, oxidative stress and the biology of ageing. Nature 408: 239-247, 2000.

35. Almeida M, Han L, Martin-Millan M, O'Brien CA and Manolagas SC: Oxidative stress antagonizes Wnt signaling in osteoblast precursors by diverting beta-catenin from $\mathrm{T}$ cell factor- to forkhead box O-mediated transcription. J Biol Chem 282: 27298-27305, 2007. 